\title{
MicroRNA-93-5p promotes the progression of human retinoblastoma by regulating the PTEN/PI3K/AKT signaling pathway
}

\author{
YONGLIANG CAO $^{1}$, FEI XIA ${ }^{1}$, PING WANG ${ }^{2}$ and MENG GAO ${ }^{1}$ \\ ${ }^{1}$ Department of Ophthalmology, Weifang Medical University; ${ }^{2}$ Department of Ophthalmology, \\ The Affiliated Hospital of Weifang Medical University, Weifang, Shandong 261000, P.R. China
}

Received February 16, 2018; Accepted September 6, 2018

DOI: $10.3892 / \mathrm{mmr} .2018 .9573$

\begin{abstract}
Numerous reports have indicated that microRNA-93-5p (miR-93-5p) is involved in the development and progression of human cancer, including non-small cell lung, gastric and breast cancer; however, the role of miR-93-5p in retinoblastoma (RB) remains unknown. In the present study, it was reported that miR-93-5p expression levels were significantly upregulated in RB tissues compared with in normal tissues by reverse transcription-quantitative polymerase chain reaction. Furthermore, it was demonstrated via cell counting kit- 8 and Transwell assays that knockdown of miR-93-5p significantly suppressed the proliferation, migration and invasion of RB cells, but promoted cellular apoptosis. Regarding the underlying mechanism, the present study reported that phosphatase and tensin homolog (PTEN) was a direct target of miR-93-5p in RB cells. Overexpression of miR-93-5p significantly inhibited the expression of PTEN; opposing results were observed when PTEN expression was downregulated. Furthermore, the present study revealed that PTEN expression levels were downregulated and were inversely correlated with that of miR-93-5p in RB tissues. Additionally, the present study demonstrated that knockdown of PTEN in miR-93-5p-depleted RB cells significantly reversed the effects of miR-93-5p on cell proliferation, migration and invasion; miR-93-5p knockdown was suggested to promote PTEN expression, consequently inhibiting the activation of phosphoinositide 3-kinase (PI3K)/protein kinase B (AKT) signaling pathway. Collectively, the results of the present study demonstrated that miR-93-5p may serve a role as an oncogene by modulating the PTEN/PI3K/AKT signaling pathway in $\mathrm{RB}$, indicating that miR-93-5p may be a potential therapeutic target for the treatment of RB.
\end{abstract}

Correspondence to: Dr Meng Gao, Department of Ophthalmology, Weifang Medical University, 7166 Baotong West Street, Weicheng, Weifang, Shandong 261000, P.R. China

E-mail: mng13333@163.com

Key words: microRNA-93-5p, retinoblastoma, proliferation, migration, phosphatase and tensin homolog/phosphoinositide 3-kinase/protein kinase B

\section{Introduction}

Retinoblastoma (RB) is the most common type of malignancy in young children and originates from immature cells within the retina (1). RB has a very high mortality rate and leads to a large number of cancer-associated deaths, particularly in developing countries (2). Increased efforts have been made in the treatment of RB in the past years; however, these developments are very limited and the 5-year survival of patients with $\mathrm{RB}$ remains quite poor (3). The majority of patients with RB are diagnosed at an advanced stage, and this is accompanied by metastasis, which is a major cause of RB-associated malignancy (4). Therefore, there is an urgent requirement to identify novel diagnostic markers and develop effective therapeutic targets for RB intervention.

MicroRNAs (miRNAs/miRs) are a class of small noncoding RNAs that regulate gene expression at the post-transcriptional level by associating with the 3'-untranslated region (UTR) of target mRNAs to accelerate their degradation $(5,6)$. Numerous reports have demonstrated that miRNAs are involved in a variety of physiological processes, including cell proliferation, development, survival and differentiation (7-10). Accumulating studies have also indicated that miRNAs may serve as oncogenes or tumor suppressors to regulate tumor cell proliferation, migration and invasion (11-14). For instance, miRNA-320a inhibits tumor proliferation and invasion by targeting c-Myc in human hepatocellular carcinoma (15). Numerous miRNAs have been reported to regulate the progression of $\mathrm{RB}$, including miR-29a (12), miR106b (13), miRNA-382 (16) and miRNA-320 (17); however, the functions of miRNAs in RB are yet to be determined and the roles of most miRNAs in RB remain unknown.

miR-93-5p has been recently reported to promote the proliferation and metastasis of numerous human cancers, including hepatocellular carcinoma (18), non-small cell lung cancer (11) and gastric cancer (19); however, the functions of miR-93-5p in RB remain elusive. In the present study, it was revealed that miR-93-5p expression was significantly upregulated in RB tissues compared with in normal tissues. Additionally, inhibition of miR-93-5p markedly suppressed the proliferation, migration and invasion while inducing cellular apoptosis of RB cells. Mechanistically, the present study demonstrated that phosphatase and tensin homolog (PTEN), an inhibitor of the phosphoinositide 3-kinase 
(PI3K)/protein kinase B (AKT) signaling pathway (20), was a target of miR-93-5p in RB cells. In addition, the expression of PTEN was downregulated and inversely correlated with that of miR-93-5p in RB cells. Furthermore, the present study revealed that knockdown of PTEN significantly rescued the proliferation, migration and invasion of RB cells transfected with miR-93-5p inhibitors. It was reported that miR-93-5p knockdown promoted PTEN expression and consequently inhibited the activation of the PI3K/AKT pathway in the present study. In conclusion, the results of the present study demonstrated that miR-93-5p may be associated with the progression of RB by modulating the PTEN/PI3K/AKT signaling pathway.

\section{Patients and methods}

Patient samples. A total of 23 human RB (16 males, 7 females; age, 15-51 years) and 12 normal retina tissue samples ( 8 males, 4 females; age, 22-61 years) were collected between February 2013 to May 2016, and were provided by the Weifang Medical University (Weifang, China). All tissue samples were harvested at surgery, immediately frozen in liquid nitrogen and stored at $-80^{\circ} \mathrm{C}$ until RNA extraction. The present study was approved by the Ethics Committee of Weifang Medical University. All of the experiments were conducted following the obtainment of written informed consent from each patient or their family.

Cellcultureandtransfection. The humanRBcellines,including HXO-Rb44, Y79 and SO-RB50, and the normal human retinal pigment epithelial cell line, ARPE-19, were purchased from the American Type Culture Collection (Manassas, VA, USA). All cancer cells were cultured in RPMI-1640 medium (Gibco; Thermo Fisher Scientific, Inc., Waltham, MA, USA) containing $100 \mathrm{IU} / \mathrm{ml}$ penicillin, $100 \mathrm{mg} / \mathrm{ml}$ streptomycin, $20 \mathrm{mM}$ glutamine and $10 \%$ heat-inactivated fetal bovine serum (FBS; Gibco; Thermo Fisher Scientific, Inc.). The normal human retinal pigment epithelial cell line ARPE-19 was cultured in Dulbecco's modified Eagles medium (DMEM; Gibco; Thermo Fisher Scientific, Inc.) with 10\% FBS, 5 mg/ml transferrin, $5 \mathrm{mg} / \mathrm{ml}$ insulin, $100 \mathrm{ng} / \mathrm{ml}$ hydrocortisone and $10 \mathrm{mM}$ HEPES. All cells were cultured in a humidified atmosphere of $5 \% \mathrm{CO}_{2}$ at $37^{\circ} \mathrm{C}$.

A total of $1 \times 10^{6}$ cells were transfected with $50 \mathrm{nM}$ miR-93-5p mimics, mimic control, miR-93-5p inhibitors, negative control (NC) inhibitor, PTEN small interfering RNA (siRNA) or control siRNA using Lipofectamine ${ }^{\circledR} 2000$ (Invitrogen; Thermo Fisher Scientific, Inc.), according to the manufacturer's protocol. miR-93-5p mimics (5'-CAAAGU GCUGUUCGUGCAGGUAG-3'), mimic control (5'-ACA UCUGCGUAAGAUUCGAGUCUA-3'), miR-93-5p inhibitors (5'-CTACCTGCACGAACAGCACTTTG-3'), NC inhibitor (5'-UCACAACCUCCUAGAAAGAGUAGA-3'),PTEN siRNA (5'-AGAUGUUAGUGACAAUGAACC-3') and control siRNA (5'-AATTCTCCGAACGTGTCACGT-3') were obtained from Shanghai GenePharma Co., Ltd. (Shanghai, China). Following transfection, cells were cultured for $48 \mathrm{~h}$ and transfection efficiency was validated using reverse transcription-quantitative polymerase chain reaction (RT-qPCR) as described below, prior to subsequent experimentation.
$R T-q P C R$. Total RNA was extracted from tumor tissue or cultured cell lines using TRIzol ${ }^{\circledR}$ reagent (Invitrogen; Thermo Fisher Scientific, Inc.), according to the manufacturer's protocols. Total RNA was reverse transcribed into cDNA using the PrimeScript ${ }^{\mathrm{TM}}$ RT reagent kit (Takara Biotechnology Co., Ltd., Dalian, China) with gDNA Eraser (Takara Biotechnology Co., Ltd.), according to the manufacturer's protocols; $1 \mathrm{mg}$ total RNA was reverse transcribed for each sample. qPCR was conducted using the TaqMan ${ }^{\mathrm{TM}}$ MicroRNA Assay kit (Applied Biosystems; Thermo Fisher Scientific, Inc.) for miRNAs and the Fast SYBR ${ }^{\mathrm{TM}}$ Green Master Mix (Applied Biosystems; Thermo Fisher Scientific Inc.) for mRNAs, on an Applied Biosystems Real-Time PCR machine (Thermo Fisher Scientific, Inc.). miR-93-5p and PTEN expression levels were normalized to U6 and GAPDH, respectively using the $2^{-\Delta \Delta C q}$ method (21). The thermocycling conditions were as follows: Denaturation at $95^{\circ} \mathrm{C}$ for $10 \mathrm{~min}$; followed by 40 cycles of denaturation at $95^{\circ} \mathrm{C}$ for $15 \mathrm{sec}$ and elongation at $60^{\circ} \mathrm{C}$ for $1 \mathrm{~min}$. The primer sequences were as follows: miR-93-5p forward, 5'-ACACTCCAGCTGGGCAAAGTGCTGTTCGTG C-3' and reverse, 5'-CTCAACTGGTGTCGTGGAGTCGGC AATTCAGTTGAGCTACCTGC-3'; PTEN forward, 5'-TCC CAGACATGACAGCCATC-3' and reverse, 5'-TGCTTTGAA TCCAAAAACCTTACT-3'; U6 forward, 5'-CTCGCTTCG GCAGCACA-3' and reverse, 5'-AACGCTTCACGAATTTGC GT-3'; and GAPDH forward, 5'-ATGTTGCAACCGGGAAGG AA-3' and reverse, 5'-AGGAAAAGCATCACCCGGAG-3'.

Cell Counting Kit-8(CCK-8) proliferation assays. Transfected cells were collected at $24 \mathrm{~h}$ post-transfection and seeded into 96-well plates at a density of $3 \times 10^{3}$ cells per well. Following culturing for 24, 48 and $72 \mathrm{~h}$, a CCK-8 assay (Beyotime Institute of Biotechnology, Shanghai, China) was performed according to the manufacturer's protocols. In brief, $10 \mu \mathrm{l}$ CCK-8 reagent was added to each well; the cells were incubated at $37^{\circ} \mathrm{C}$ with $5 \% \mathrm{CO}_{2}$ for $2 \mathrm{~h}$. Absorbance was determined at a wavelength of $450 \mathrm{~nm}$ using an ELx808 absorbance reader (BioTek Instruments, Inc., Winooski, VT, USA). Each assay was performed in triplicate and repeated three times.

Transwell assays. Transwell chamber assays were performed to analyze cell invasion and migration. Matrigel was employed to pre-coat the membrane of Transwell chambers to simulate a matrix barrier for the invasion assay. The transfected cells in the $\log$ phase were seeded on the upper champers at a density of $2 \times 10^{5}$ cells/well in $200 \mu 1$ serum-free RPMI-1640 medium. A total of $600 \mu \mathrm{l}$ RPMI-1640 medium with $10 \%$ FBS was added to the lower chamber to stimulate cell migration and invasion. Following $24 \mathrm{~h}$ of incubation at $37^{\circ} \mathrm{C}$, cells that had migrated or invaded to the lower chamber were fixed with paraformaldehyde for $5 \mathrm{~min}$ at $25^{\circ} \mathrm{C}$ and stained with $0.1 \%$ crystal violet for $5 \mathrm{~min}$ at $25^{\circ} \mathrm{C}$. The images of cells were obtained under a TS100 inverted light microscope (Nikon Corporation, Tokyo, Japan) at magnification, x200; the cell number was counted in five selected randomly fields per membrane.

Western blot analysis. Y79 and SO-RB50 cells were lysed in cold radioimmunoprecipitation assay buffer, and protein concentration was determined using a Pierce ${ }^{\mathrm{TM}}$ BCA Protein Assay kit (both Thermo Fisher Scientific, Inc.). Protein (40 $\mu \mathrm{g} / \mathrm{lane})$ 

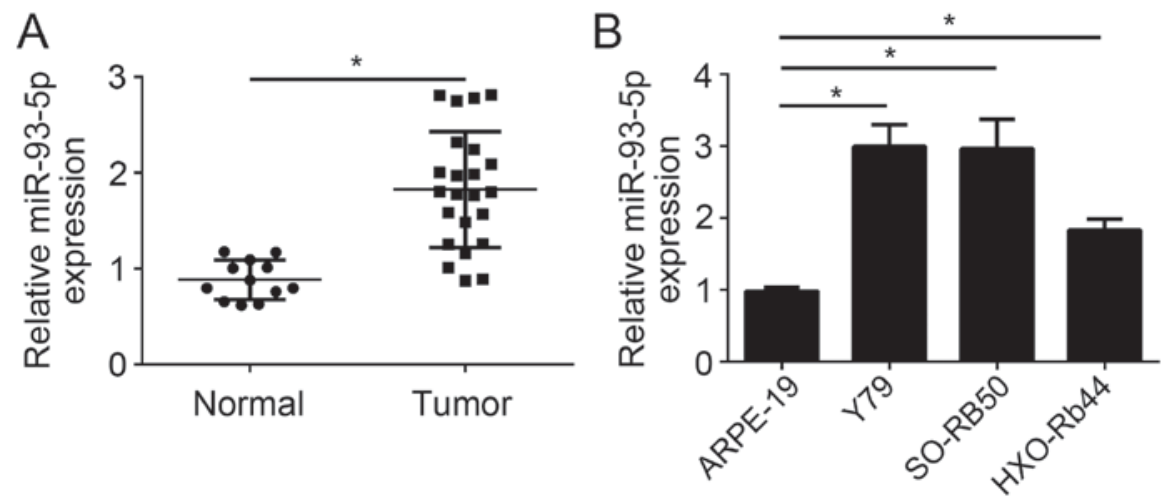

Figure 1. miR-93-5p is highly expressed in RB tissues. (A) Relative expression levels of miR-93-5p in RB tissues and normal tissues were determined by RT-qPCR. (B) RT-qPCR analysis revealed that miR-93-5p expression levels were upregulated in RB cell lines compared with in ARPE-19 cells. ${ }^{*} \mathrm{P}<0.05$ vs. normal tissues or ARPE-19 cells. RB, retinoblastoma; miR, microRNA; RT-qPCR, reverse transcription-quantitative polymerase chain reaction.

was separated via $10 \%$ SDS-PAGE and then transferred to a polyvinylidene difluoride (PVDF) membrane (Thermo Fisher Scientific, Inc.). The membrane was blocked using $5 \%$ non-fat milk in PBS (Thermo Fisher Scientific, Inc.) containing 0.1\% Tween-20 (Sigma-Aldrich; Merck KGaA, Darmstadt, Germany) at room temperature for $2 \mathrm{~h}$. Subsequently, the PVDF membrane was incubated with rabbit anti-PTEN (1:1,000; cat. no. ab32199; Abcam, Cambridge, MA, USA), rabbit anti-phosphorylated (p)-AKT (1:1,000; cat. no. 4060; Cell Signaling Technology, USA), rabbit anti-AKT (1:1,000; cat. no. ab18785) and rabbit anti-GAPDH (1:1,000; cat. no. ab9485; both Abcam) primary antibodies at room temperature for $2 \mathrm{~h}$. Following washing with PBS for 10 min, the PVDF membrane was incubated with horseradish peroxidase-conjugated goat anti-rabbit secondary antibodies (1:5,000; cat. no. ab7090; Abcam) at room temperature for $1 \mathrm{~h}$. Membranes were then washed with PBS for $10 \mathrm{~min}$ and the protein bands were visualized using the Pierce ${ }^{\mathrm{TM}} \mathrm{ECL}$ Western Blotting Substrate kit (Thermo Fisher Scientific, Inc.), according to the manufacturer's protocol. Protein densitometry was performed using ImageJ Software version 1.41 (National Institutes of Health, Bethesda, MD, USA).

Cell apoptosis assays. Cell apoptosis was determined using an Annexin V-fluorescein isothiocyanate (FITC) Apoptosis Detection kit (Nanjing KeyGen Biotech Co., Ltd., Nanjing, China), according to the manufacturer's protocols. Briefly, $\mathrm{RB}$ cells were harvested $48 \mathrm{~h}$ following transfection and then washed with PBS. Then, $5 \times 10^{4}$ cells were resuspended in $500 \mu \mathrm{l}$ of binding buffer containing $5 \mu \mathrm{l}$ of Annexin V-FITC and $5 \mu \mathrm{l}$ of propidium iodide (PI) for $10 \mathrm{~min}$ at $4^{\circ} \mathrm{C}$. Subsequently, apoptosis was determined using a flow cytometer. The data were analyzed with FlowJo software version 5.7.2 (FlowJo LLC, Ashland, OR, USA). The total percentage Annexin V-positive cells indicated apoptotic cells.

Luciferase assay. TargetScan version 7.1 (http://www. targetscan.org/index.html) was used to predict potential miR-93-5p targets. The wild-type (WT) 3'-UTR sequence of PTEN or the mutant (Mut) 3'-UTR sequence of PTEN was amplified by PCR and incorporated into the pGL3 control vector (Promega Corporation, Madison, WI, USA) between $M l u \mathrm{I}$ and $X h o \mathrm{I}$ restriction sites, to obtain the WT-PTEN-3'-UTR or Mut-PTEN-3'-UTR, respectively. A total of $2 \times 10^{4}$ cells/well were seeded into 24-well plates the day prior to transfection and then cells were cotransfected with $1 \mathrm{mg}$ WT or Mut PTEN-3'-UTR along with $50 \mathrm{nM}$ miR-93-5p mimics or mimic control using Lipofectamine ${ }^{\circledR} 2000$ (Invitrogen; Thermo Fisher Scientific, Inc.). At $48 \mathrm{~h}$ following cotransfection, the luciferase activity for the WT or Mut PTEN 3'-UTR was measured using the Dual-Luciferase ${ }^{\circledR}$ Reporter Assay system (Promega Corporation) and normalized to Renilla luciferase activity.

Statistical analysis. SPSS statistical software for Windows version 19.0 (IBM Corp., Armonk, NY, USA) and GraphPad Prism version 5.01 (GraphPad Software, Inc., La Jolla, CA, USA) software were used for statistical analysis. All experiments were repeated at least three times and data are represented as the mean \pm standard deviation from at least three independent experiments. A Student's t-test and one-way analysis of variance followed by a Tukey's post hoc test was used to compare two or multiple groups, respectively, for statistical significance. Spearman's rank correlation analysis was performed to analyze correlation between miR-93-5p and PTEN expression levels. $\mathrm{P}<0.05$ was considered to indicate a statistically significant difference.

\section{Results}

miR-93-5p is highly expressed in RB tissues and cell lines. The present study analyzed the expression levels of miR-93-5p in RB tissues by RT-qPCR. It was observed that the expression levels of miR-93-5p were significantly upregulated in RB tissues $(n=23)$ compared with in normal tissues $(n=12)$ $(\mathrm{P}<0.05$; Fig. 1A). Furthermore, RT-qPCR analysis also indicated that miR-93-5p expression levels were also significantly upregulated in the RB cell lines, including Y79, SO-RB50 and HXO-Rb44, compared with in ARPE-19 cells $(\mathrm{P}<0.05$; Fig. 1B). miR-93-5p expression levels were highest in Y79 and SO-RB50 cells; therefore, these two cell lines were selected for following experiments. These results demonstrated that miR-93-5p expression was upregulated in RB cells, indicating that miR-93-5p may be involved in the progression of RB.

Knockdown of miR-93-5p inhibits $R B$ cell proliferation and promotes apoptosis. To investigate the function of miR-93-5p, the expression of miR-93-5p was downregulated in Y79 and 
A

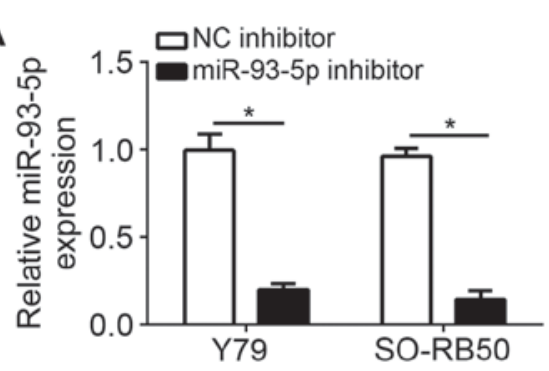

B

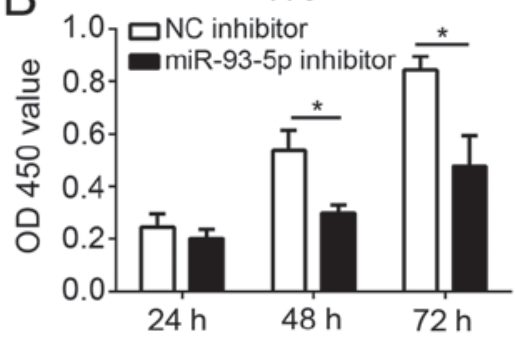

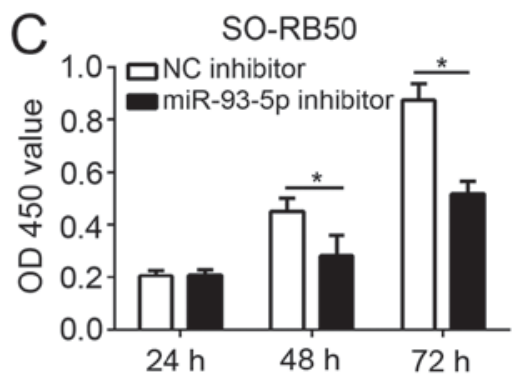

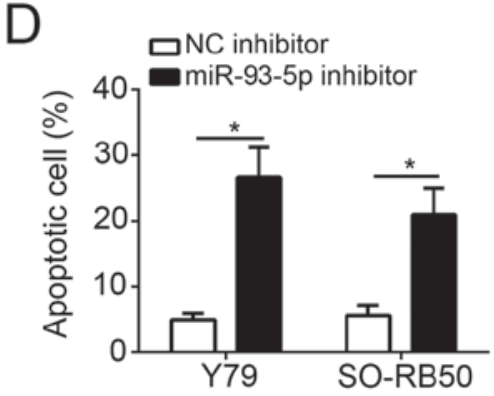

NC inhibitor miR-93-5p inhibitor

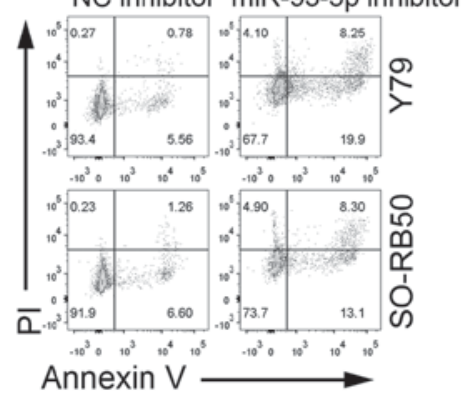

Figure 2. Knockdown of miR-93-5p inhibits retinoblastoma cell proliferation and promotes apoptosis. (A) Relative expression of miR-93-5p in Y79 and SO-RB50 cells transfected with miR-93-5p or NC inhibitors. Cell Counting kit-8 assays were used to measure the proliferation of (B) Y79 and (C) SO-RB50 cells. (D) Flow cytometric analysis indicated that knockdown of miR-93-5p promoted the apoptosis of Y79 and SO-RB50 cells. The percentage of apoptotic cells indicates the total percentage of Annexin V-positive cells. "P<0.05 vs. NC inhibitor. OD, optical density; miR, microRNA; NC, negative control; PI, propidium iodide.

SO-RB50 cells via transfection with a miR-93-5p inhibitor. RT-qPCR analysis indicated that miR-93-5p expression levels were significantly downregulated in Y79 and SO-RB50 cells compared with in cells transfected with the $\mathrm{NC}$ inhibitor ( $\mathrm{P}<0.05$; Fig. 2A). In addition, CCK-8 assays were performed to analyze cell proliferation. As presented in Fig. 2B and C, miR-93-5p knockdown significantly suppressed the proliferation of Y79 and SO-RB50 cells at 48 and $72 \mathrm{~h}$ following transfection compared with in the $\mathrm{NC}$ inhibitor group $(\mathrm{P}<0.05)$. Furthermore, cell apoptosis was determined by Annexin V/PI staining. The results demonstrated that miR-93-5p knockdown significantly increased the percentage of Y79 and SO-RB50 apoptotic cells $(\mathrm{P}<0.05$; Fig. 2D). The results suggested that miR-93-5p promoted the malignant behavior of RB cells.

miR-93-5p knockdown suppresses $R B$ cell migration and invasion. To analyze the effects of miR-93-5p on tumor metastasis, the cell migration and invasive abilities of RB cells transfected with miR-93-5p or control inhibitors were investigated via Transwell assays. The results indicated that miR-93-5p knockdown significantly suppressed the migration and invasive abilities of Y79 and SO-RB50 cells compared with in the $\mathrm{NC}$ inhibitor group $(\mathrm{P}<0.05$; Fig. $3 \mathrm{~A}$ and $\mathrm{B})$.

PTEN is a target of miR-93-5p. To further investigate the mechanism of miR-93-5p in RB cells, bioinformatics analysis was conducted. The present study reported that PTEN was a potential target of miR-93-5p. A potential binding site of miR-93-5p was determined in the 3'-UTR of PTEN mRNA (Fig. 4A). To verify this prediction, luciferase reporter assays were conducted with RB cells cotransfected with miR-93-5p mimics or control and WT-PTEN-3'-UTR or Mut-PTEN-3'-UTR. Firstly, RT-qPCR was performed to confirm successful miR-93-5p overexpression
(Fig. 4B and C). Next, the luciferase reporter assay revealed that miR-93-5p overexpression significantly inhibited the luciferase activity of the WT-PTEN-3'-UTR compared with in cells cotransfected with control $(\mathrm{P}<0.05)$; however, that of the Mut-PTEN-3'-UTR in Y79 and SO-RB50 cells were unaffected (Fig. 4B and C). To directly analyze the effects of miR-93-5p on PTEN expression, miR-93-5p mimic or control was transfected into RB cells, which demonstrated that overexpression of miR-93-5p significantly reduced the mRNA expression levels of PTEN in Y79 and SO-RB50 cells (Fig. 4D). Consistently, knockdown of miR-93-5p markedly upregulated the protein expression levels of PTEN in Y79 and SO-RB50 cells (Fig. 4E). The data demonstrated that PTEN may be a direct target of miR-93-5p in RB cells.

PTEN expression is inversely correlated with miR-93-5p expression in $R B$ tissues. The present study investigated the expression levels of PTEN in RB tissues by RT-qPCR. The results revealed that PTEN expression levels were significantly downregulated in RB tissues compared with in normal tissues (Fig. 5A). In addition, a statistically significant inverse correlation was observed via Spearman's correlation analysis between miR-93-5p and PTEN levels in RB tissues (Fig. 5B).

PTEN knockdown reverses the inhibitory effects of miR-93-5p depletion on the proliferation, migration and invasion of $R B$ cells. As miR-93-5p was determined to be significantly overexpressed in tumor cells (Fig. 1B), a rescue assay using miR-93-5p inhibitors was conducted; the expression levels of PTEN were downregulated in miR-93-5p-depleted RB cells via transfection with a specific siRNA against PTEN. RT-qPCR confirmed the efficiency of PTEN siRNA transfection (Fig. 6A). Western blotting indicated that the expression 
A

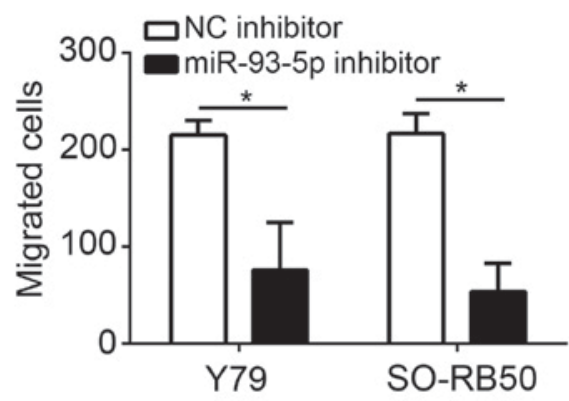

$\mathrm{B}$

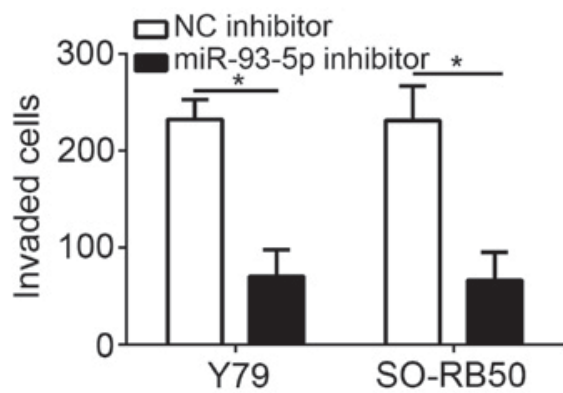

NC inhibitor miR-93-5p inhibitor

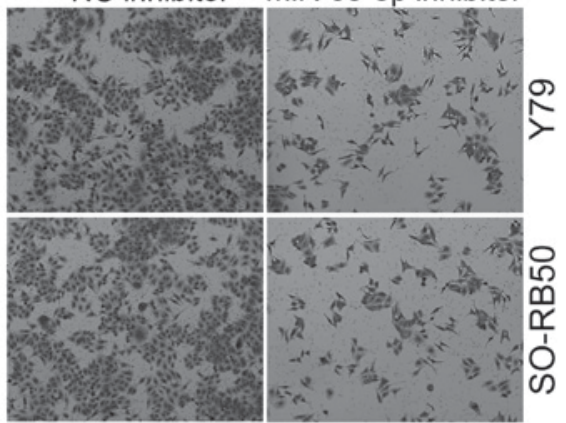

NC inhibitor miR-93-5p inhibitor

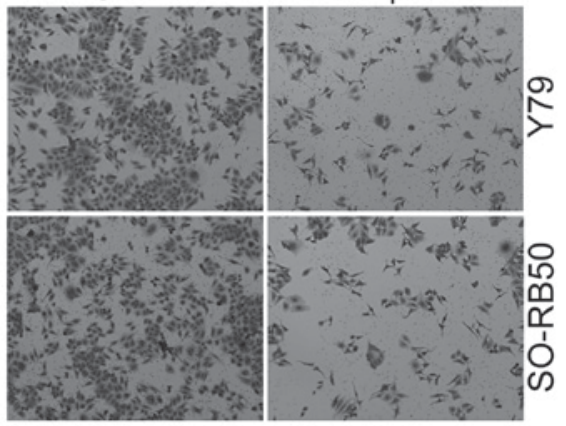

Figure 3. miR-93-5p downregulation suppresses RB cell migration and invasion. (A) Cell migration was determined via a Transwell migration assay of RB cells transfected with miR-93-5p or an NC inhibitor. (B) Cell invasion was detected via a Transwell invasion assay of RB cells transfected with an miR-93-5p inhibitor or control. " $\mathrm{P}<0.05$ vs. $\mathrm{NC}$ inhibitor. $\mathrm{NC}$, negative control; miR, microRNA; RB, retinoblastoma.

A

miR-93-5p: 3'-gauggacgugcuuguCGUGAAAC-5 | || ||| PTEN-3'UTR: 5'-gauuaauaaagaugGCACUUUc-3' PTEN-3'UTR: 5'-gauuaauaaagaugCGUGAAAC-3' Mutation
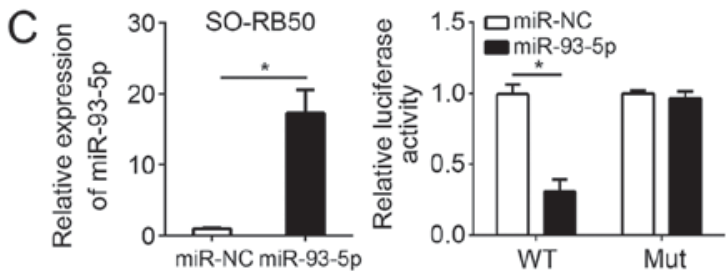
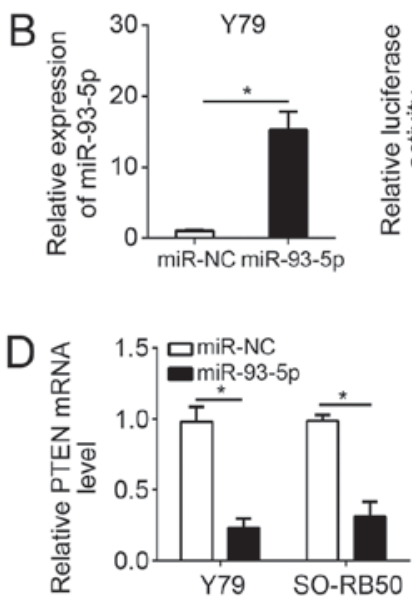
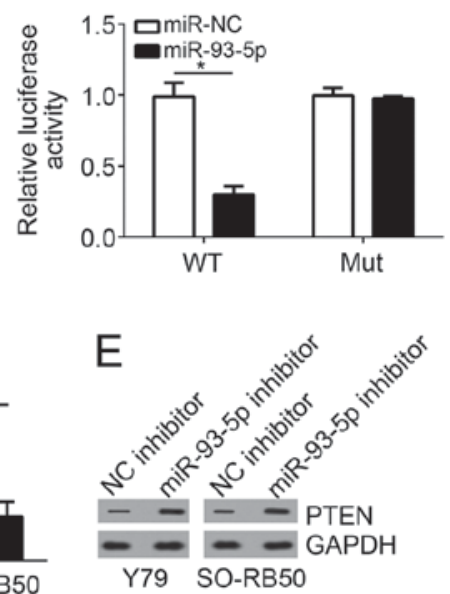

Figure 4. PTEN is a target of miR-93-5p. (A) Predicted binding site of miR-93-5p in the 3'-UTR region of PTEN mRNA. Luciferase reporter assay was performed in (B) Y79 and (C) SO-RB50 cells transfected with the WT or Mut-PTEN-3'UTR reporter plasmid and miR-93-5p mimics or miR-NC. (D) Reverse transcription-quantitative polymerase chain reaction analysis indicated that miR-93-5p overexpression inhibited the mRNA level of PTEN in Y79 and SO-RB50 cells. (E) Western blotting demonstrated that miR-93-5p knockdown increased the protein levels of PTEN in Y79 and SO-RB50 cells. "P<0.05 vs. NC group. Mut, mutant; miR, microRNA; NC, negative control; PTEN, phosphatase and tensin homolog; UTR, untranslated region; WT, wild type.

levels of PTEN were markedly downregulated compared with in miR-93-5p-downregulated RB cells (Fig. 6A). Notably, knockdown of PTEN significantly rescued the suppressive effects of miR-93-5p inhibition on RB cell proliferation, migration and invasion $(\mathrm{P}<0.05$; Fig. 6B-D). These results indicated that miR-93-5p may exerts its oncogenic roles within RB cells by regulating PTEN expression, at least partly.

miR-93-5p knockdown suppresses the PI3K/AKT signaling pathway in RB cells. PTEN has been demonstrated to be a negative regulator of the PI3K/AKT signaling pathway, which is abnormally activated in numerous types of tumors, including RB (22). The present study investigated whether miR-93-5p may exert an effect on the PI3K/AKT signaling pathway. Western blotting results indicated that knockdown of miR-93-5p notably upregulated the protein levels of PTEN and inhibited that of p-AKT compared with in the control group (Fig. 7A and B), which suggested that miR-93-5p positively activates the PI3K/AKT signaling pathway in RB cells. In conclusion, the present study demonstrated that miR-93-5p 

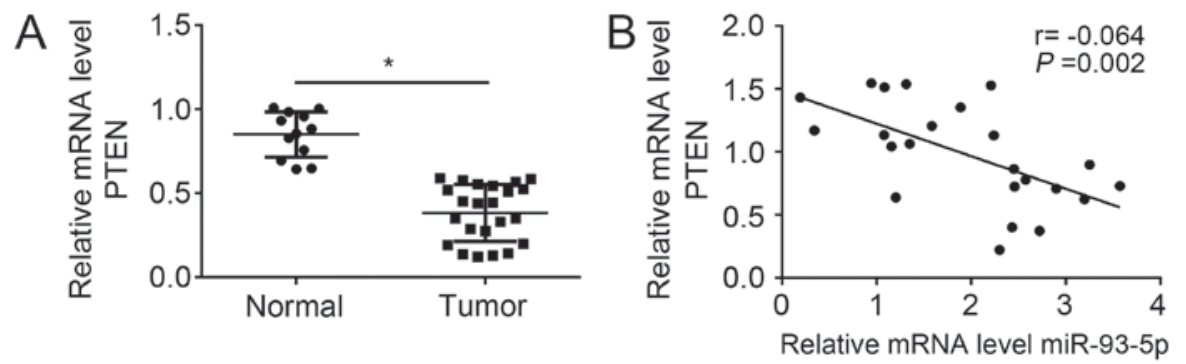

Figure 5. PTEN expression is inversely correlated with miR-93-5p expression in RB tissues. (A) PTEN mRNA expression levels were determined in normal retinal and RB tumor samples by reverse transcription-quantitative polymerase chain reaction. (B) Relative inverse correlation between PTEN and miR-93-5p expression as determined by Spearman's correlation analysis of RB tissues. " $\mathrm{P}<0.05$ vs. normal group. PTEN, phosphatase and tensin homolog; miR, microRNA; RB, retinoblastoma.
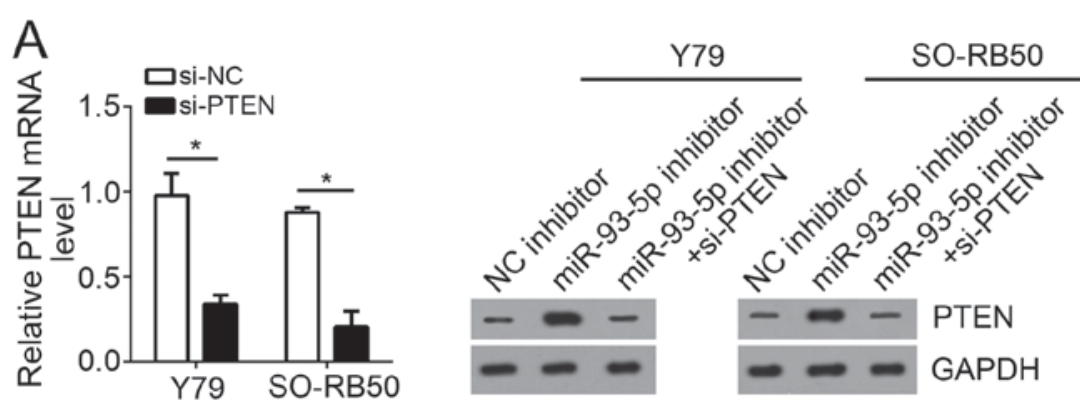

B 口NC inhibitor
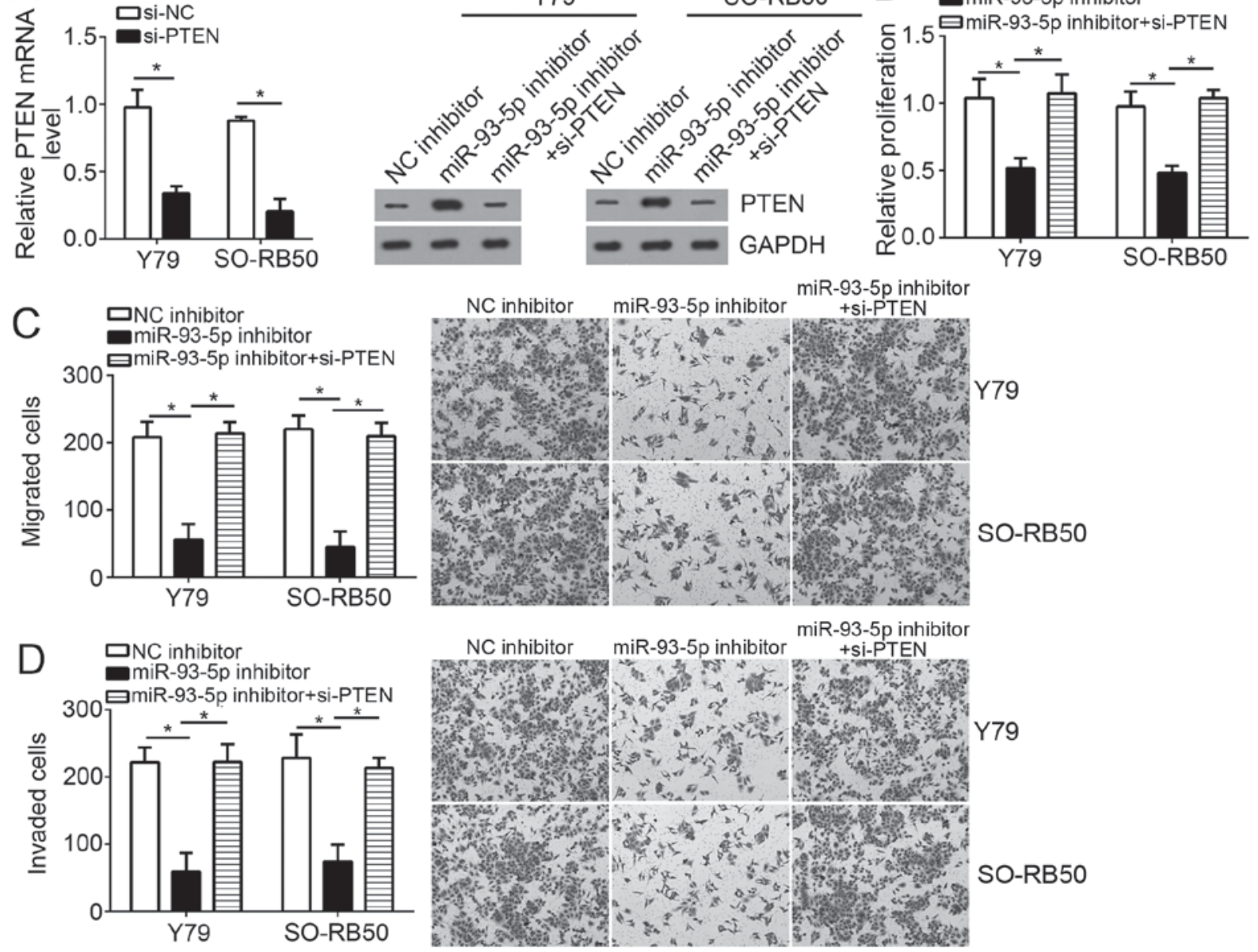

Figure 6. PTEN knockdown reverses the inhibitory effect of miR-93-5p depletion on proliferation, migration and invasion in RB cells. (A) PTEN mRNA and protein expression levels were measured by reverse transcription-quantitative polymerase chain reaction and western blotting in RB cells transfected with miR-93-5p inhibitors in the presence or absence of PTEN siRNA. Analysis was conducted $72 \mathrm{~h}$ following transfection. GAPDH was used as an internal control. (B) Cell proliferation of RB cells $72 \mathrm{~h}$ following transfection with an miR-93-5p inhibitor in the presence of a PTEN siRNA as determined by a Cell Counting Kit-8. (C) Cell migration and (D) invasion was determined via Transwell assays of RB cells $48 \mathrm{~h}$ following transfection with miR-93-5p inhibitors in the presence or absence of a PTEN siRNA. "P<0.05 vs. NC group. PTEN, phosphatase and tensin homolog; miR, microRNA; RB, retinoblastoma; siRNA, small interfering RNA.

may exert an oncogenic role in $\mathrm{RB}$ via regulation of the PTEN/PI3K/AKT signaling pathway.

\section{Discussion}

In the past decade, miRNAs have been widely demonstrated to serve essential functions in the initiation and progression of $\mathrm{RB}$ via regulating the expression of specific genes (23). For instance, Liu et al (24) reported that the proliferation, migration and invasion of human RB cells were significantly suppressed by miR-124 in a signal transducer and activator of transcription 3-dependent manner. Lei et al (25) reported that miR-101 was downregulated in RB tissues and suppressed tumor cell growth and proliferation by inhibiting the expression of enhancer of 

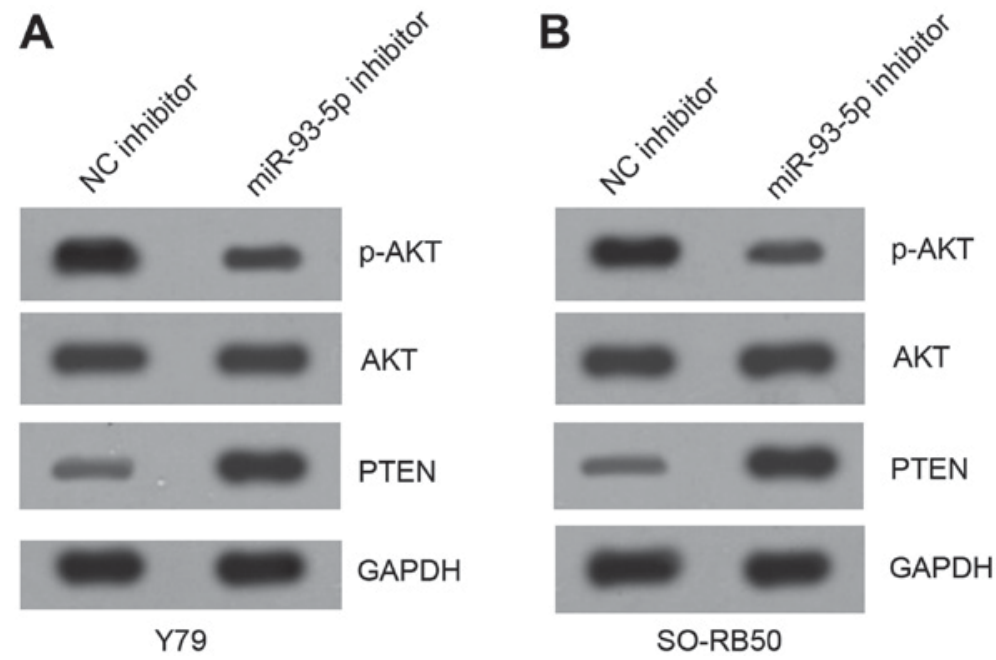

Figure 7. miR-93-5p knockdown suppressed the PI3K/AKT signaling pathway in retinoblastoma cells. Western blot analysis indicated that miR-93-5p knockdown inhibited the activation of PI3K/AKT signaling pathway in (A) Y79 and (B) SO-RB50 cells. AKT, protein kinase B; miR, microRNA; NC, negative control; p, phosphorylated; PTEN, phosphatase and tensin homolog.

zeste homolog 2. Liu et al (26) demonstrated that miR-34a was downregulated in RB tissues and enhanced tumor cell chemosensitivity and promoted cell death by targeting high mobility group box 1 to suppress autophagy. Wang et al (27) reported that miRNA-183 inhibited the proliferation, migration and invasion of RB cells downregulating the expression of low-density lipoprotein receptor-related protein 6. Martin et al (28) revealed that miR-449 significantly inhibited the proliferation and induced the apoptosis of RB cells. Additionally, Wang et al (29) reported that miR-365b-3p was downregulated in RB tissues and was associated with cell cycle arrest, and enhanced tumor cell apoptosis by targeting paired box protein Pax-6. In the present study, it was observed that miR-93-5p was significantly upregulated in RB tissues and cell lines. Functional experiments demonstrated that miR-93-5p knockdown inhibited the proliferation, migration and invasion, but induced the apoptosis of RB cells. The results of the present study indicated that miR-93-5p serves a crucial role in the progression of RB.

miR-93-5p has been demonstrated to serve as an oncogene and promote tumor growth and metastasis in numerous types of cancer, including ovarian carcinoma (30), endometrial carcinoma (31), triple-negative breast cancer (32), gastric cancer (19), non-small cell lung cancer (11) and hepatocellular carcinoma (18). However, to the best of our knowledge, the physiological functions and underlying molecular mechanism of miR-93-5p in RB have not been investigated. In the present study, it was reported that miR-93-5p expression was significantly upregulated in RB specimens compared with in normal retinal tissues. Consistently, the expression levels of miR-93-5p were higher in RB cell lines than that of ARPE-19 cells. Additionally, the present study revealed that knockdown of miR-93-5p suppressed the proliferation, migration and invasion of RB cells, but enhanced cellular apoptosis. These results suggested that miR-93-5p serves as an oncogene in RB.

Increasing evidence has indicated that miRNAs exert biological functions in cancer cells by suppressing the expression of target genes $(31,33)$. Bioinformatics analysis using TargetScan indicated that PTEN may be a potential target of miR-93-5p as determined in the present study. In addition, the interaction between PTEN and miR-93-5p in RB cells was demonstrated by luciferase reporter assays, RT-qPCR and western blot analysis in the present study. PTEN has been reported to suppress tumor growth and metastasis in various cancers by regulating proliferation, migration and invasion, and the cell cycle (20). Importantly, accumulating evidence has indicated that PTEN is a negative regulator of the PI3K/AKT signaling pathway, in which aberrant activation leads to the progression of several types of cancer, such as RB (16,34); however, the mechanism underlying the regulation of PTEN in $\mathrm{RB}$ requires further investigation. In the present study, the inhibition of miR-93-5p was associated with significantly enhanced PTEN expression in RB cells; opposing results were observed when PTEN expression was downregulated. In addition, the present study reported that miR-93-5p expression levels were inversely correlated with PTEN mRNA expression in RB tissues. Knockdown of PTEN partially reversed the effects of miR-93-5p depletion on RB cell proliferation, migration and invasion. Additionally, the results of the present study also demonstrated that knockdown of miR-93-5p inhibited the activation of the PI3K/AKT pathway in RB cells. These observations suggest that miR-93-5p exhibited an oncogenic role in $\mathrm{RB}$ via the targeting of PTEN. Compared to the previous study by Wei et al (34), the present study revealed the relationship between PTEN and miR-93-5p in RB progression.

In conclusion, the present study reported that miR-93-5p may promoted the progression of $\mathrm{RB}$ by regulating cell proliferation, apoptosis, migration and invasion in a PTEN/PI3K/AKT signaling pathway-dependent manner. These results suggest that miR-93-5p may be a potential therapeutic target for the treatment of RB; however, further investigation is required.

\section{Acknowledgements}

Not applicable.

\section{Funding}

No funding was received. 


\section{Availability of data and materials}

All data generated or analyzed during this study are included in this published article.

\section{Authors' contributions}

YC and MG made substantial contributions to the design of the present study, analyzed and interpreted the results, and wrote the manuscript. FX and PW performed the experiments, including apoptosis detection and luciferase reporter assays. All authors read and approved the final manuscript.

\section{Ethics approval and consent to participate}

For the use of human samples, the present study was approved by the Institutional Ethics Committee of Weifang Medical University and all enrolled patients provided written informed consent.

\section{Patient consent for publication}

Not applicable.

\section{Competing interests}

The authors declare that they have no competing interests.

\section{References}

1. Shields CL and Shields JA: Retinoblastoma management: Advances in enucleation, intravenous chemoreduction, and intra-arterial chemotherapy. Curr Opin Ophthalmol 21: 203-212, 2010.

2. Jabbour P, Chalouhi N, Tjoumakaris S, Gonzalez LF, Dumont AS, Chitale R, Rosenwasser R, Bianciotto CG and Shields C: Pearls and pitfalls of intraarterial chemotherapy for retinoblastoma. J Neurosurg Pediatr 10: 175-181, 2012.

3. Houston SK, Murray TG, Wolfe SQ and Fernandes CE: Current update on retinoblastoma. Int Ophthalmol Clin 51: 77-91, 2011.

4. Kivelä T: The epidemiological challenge of the most frequent eye cancer: Retinoblastoma, an issue of birth and death. $\mathrm{Br}$ J Ophthalmol 93: 1129-1131, 2009.

5. Chen K and Rajewsky N: The evolution of gene regulation by transcription factors and microRNAs. Nat Rev Genet 8: 93-103, 2007.

6. Du T and Zamore PD: Beginning to understand microRNA function. Cell Res 17: 661-663, 2007.

7. Chen CZ, Li L, Lodish HF and Bartel DP: MicroRNAs modulate hematopoietic lineage differentiation. Science 303: 83-86, 2004.

8. Brennecke J, Hipfner DR, Stark A, Russell RB and Cohen SM bantam encodes a developmentally regulated microRNA that controls cell proliferation and regulates the proapoptotic gene hid in Drosophila. Cell 113: 25-36, 2003.

9. Geng S and Zhou W: Influence of extrinsic inputs and synaptic gains on dynamics of Wendling's neural mass model: A bifurcation analysis. J Integr Neurosci 15: 463-483, 2016.

10. $\mathrm{Xu}$ P, Vernooy SY, Guo M and Hay BA: The Drosophila microRNA Mir-14 suppresses cell death and is required for normal fat metabolism. Curr Biol 13: 790-795, 2003.

11. Yang W, Bai J, Liu D, Wang S, Zhao N, Che R and Zhang H: MiR-93-5p up-regulation is involved in non-small cell lung cancer cells proliferation and migration and poor prognosis. Gene 647: 13-20, 2018.

12. Liu S, Zhang X, Hu C, Wang Y and Xu C: miR-29a inhibits human retinoblastoma progression by targeting STAT3. Oncol Rep 39: 739-746, 2018.

13. Yang G, Fu Y, Zhang L, Lu X and Li Q: miR106b regulates retinoblastoma Y79 cells through Runx3. Oncol Rep 38: 3039-3043, 2017.
14. Shen Y, Ye YF, Ruan LW, Bao L, Wu MW and Zhou Y: Inhibition of miR-660-5p expression suppresses tumor development and metastasis in human breast cancer. Genet Mol Res 16, 2017.

15. Xie F, Yuan Y, Xie L, Ran P, Xiang X, Huang Q, Qi G, Guo X, Xiao $\mathrm{C}$ and Zheng S: miRNA-320a inhibits tumor proliferation and invasion by targeting c-Myc in human hepatocellular carcinoma. Onco Targets Ther 10: 885-894, 2017.

16. Song D, Diao J, Yang Y and Chen Y: MicroRNA382 inhibits cell proliferation and invasion of retinoblastoma by targeting BDNF-mediated PI3K/AKT signalling pathway. Mol Med Rep 16: 6428-6436, 2017.

17. Zhao Y, Zhang S and Zhang Y: MicroRNA-320 inhibits cell proliferation, migration and invasion in retinoblastoma by targeting specificity protein 1. Mol Med Rep 16: 2191-2198, 2017.

18. Wang X, Liao Z, Bai Z, He Y, Duan J and Wei L: MiR-93-5p promotes cell proliferation through down-regulating PPARGC1A in hepatocellular carcinoma cells by bioinformatics analysis and experimental verification. Genes (Basel) 9: E51, 2018.

19. Li L, Zhao J, Huang S, Wang Y, Zhu L, Cao Y, Xiong J and Deng J: MiR-93-5p promotes gastric cancer-cell progression via inactivation of the Hippo signaling pathway. Gene 641: 240-247, 2018.

20. Waniczek D, Śnietura M, Lorenc Z, Nowakowska-Zajdel E and Muc-Wierzgoń M: Assessment of PI3K/AKT/PTEN signaling pathway activity in colorectal cancer using quantum dot-conjugated antibodies. Oncol Lett 15: 1236-1240, 2018.

21. Livak KJ and Schmittgen TD: Analysis of relative gene expression data using real-time quantitative PCR and the 2(-Delta Delta C(T)) method. Methods 25: 402-408, 2001

22. Di Fiore R, Drago-Ferrante R, D'Anneo A, Augello G, Carlisi D, De Blasio A, Giuliano M, Tesoriere G and Vento R: In human retinoblastoma Y79 cells okadaic acid-parthenolide co-treatment induces synergistic apoptotic effects, with PTEN as a key player. Cancer Biol Ther 14: 922-931, 2013.

23. Yang $Y$ and Mei Q: miRNA signature identification of retinoblastoma and the correlations between differentially expressed miRNAs during retinoblastoma progression. Mol Vis 21: 1307-1317, 2015.

24. Liu S, Hu C, Wang Y, Shi G, Li Y and Wu H: miR-124 inhibits proliferation and invasion of human retinoblastoma cells by targeting STAT3. Oncol Rep 36: 2398-2404, 2016.

25. Lei Q, Shen F, Wu J, Zhang W, Wang J and Zhang L: MiR-101, downregulated in retinoblastoma, functions as a tumor suppressor in human retinoblastoma cells by targeting EZH2. Oncol Rep 32: 261-269, 2014.

26. Liu K, Huang J, Xie M, et al: MIR34A regulates autophagy and apoptosis by targeting HMGB1 in the retinoblastoma cell. Autophagy 10: 442-452, 2014.

27. Wang J, Wang X, Li Z, Liu H and Teng Y: MicroRNA-183 suppresses retinoblastoma cell growth, invasion and migration by targeting LRP6. FEBS J 281: 1355-1365, 2014.

28. Martin A, Jones A, Bryar PJ, et al: MicroRNAs-449a and $-449 \mathrm{~b}$ exhibit tumor suppressive effects in retinoblastoma. Biochem Biophys Res Commun 440: 599-603, 2013.

29. Wang J, Wang X, Wu G, Hou D and Hu Q: MiR-365b-3p, down-regulated in retinoblastoma, regulates cell cycle progression and apoptosis of human retinoblastoma cells by targeting PAX6. FEBS Lett 587: 1779-1786, 2013.

30. Chen X, Chen S, Xiu YL, Sun KX, Zong ZH and Zhao Y: RhoC is a major target of microRNA-93-5P in epithelial ovarian carcinoma tumorigenesis and progression. Mol Cancer 14: 31, 2015.

31. Chen S, Chen X, Sun KX, Xiu YL, Liu BL, Feng MX, Sang XB and Zhao Y: MicroRNA-93 promotes epithelial-mesenchymal transition of endometrial carcinoma cells. PLoS One 11: e0165776, 2016.

32. Shyamasundar S, Lim JP and Bay BH: miR-93 inhibits the invasive potential of triple-negative breast cancer cells in vitro via protein kinase WNK1. Int J Oncol 49: 2629-2636, 2016.

33. Catela Ivkovic T, Voss G, Cornella H and Ceder Y: microRNAs as cancer therapeutics: A step closer to clinical application. Cancer Lett 407: 113-122, 2017.

34. Wei D, Miao Y, Yu L, Wang D and Wang Y: Downregulation of microRNA198 suppresses cell proliferation and invasion in retinoblastoma by directly targeting PTEN. Mol Med Rep 18: 595-602, 2018 